\title{
Deutsche Entlehnungen (Substantive) im Tschechischen aus stilistischer Sicht
}

\author{
Mojmír Muzikant
}

\section{Einleitung}

Das Ziel des Aufsatzes ist es, den zu behandelnden Gegenstand vielmehr klassifizierend darzustellen als über die Ursachen und Wurzeln dieser Erscheinung Überlegungen anzustellen. Aus historischer Sicht hat sich dieser Thematik eine Reihe von bedeutenden Germanisten und Bohemisten angenommen. In jüngster Zeit sind in diesem Fachbereich zwei erwähnenswerte Arbeiten entstanden. Eine hinsichtlich der Tiefe und Breite des zu verarbeitenden Sprachmaterials imponierende Beschreibung der deutschen Entlehnungen im Tschechischen und Slowakischen in Bezug auf deren Ursprung und historische Entwicklung stellt NEWERKLA (2004) vor. Eine Untersuchung der Germanismen im nichtschriftsprachlichen Tschechischen hat in jüngster Zeit MIČKA (2007) in seiner Diplomarbeit unternommen. Als Quelle für die Erarbeitung hat er HUGO (2006) herangezogen. Wegen der zu großen Belegmenge hat er die Auswahl auf die Buchstaben A bis $\mathrm{F}$ beschränkt. Den übernommenen Wortbestand an Germanismen hat er mit Rücksicht auf die lexikalische Semantik in zwölf Gruppen eingeteilt. Diese hat er dann auf ihre Vorkommenshäufigkeit überprüft, indem er die exzerpierten Germanismen mit drei Korpora der tschechischen Sprache verglich (SYN 2000, BMK, PMK - unter http://ucnk.ff.cuni.cz abrufbar). Der Vergleich machte es möglich, Angaben über die territoriale Bestimmung und Häufigkeit der einzelnen Lexeme bzw. der ganzen semantisch festgelegten Wortgruppen zu präzisieren. Die Frage der stilistischen Merkmale als Kriterium für die Binnendifferenzierung von deutschen Entlehnungen im Tschechischen bleibt unerwähnt. Bei flüchtiger Durchsicht der vorhandenen Wortlisten fallen jedoch auch gewisse Klassifizierungsdefizite auf. So behauptet der Autor z. B. auf S. 44, dass ferman im Sinne, denní vývěska o rozvrhu práce auf das deutsche Ausgangsformativ Fahrplan zurückgeht. Wenn er jedoch im Wörterbuch der tschechischen Schriftsprache nachgeschlagen hätte, hätte er dieses Wort nicht zu Germanismen zählen können. Das Wort kommt nämlich aus dem Persischen und hat im Soziolekt der Beamten die Bedeutung, Erlass, Anordnung, Befehl, Bekanntmachung, Rundschreiben' (SSJČ I: 1989, 492). Dieser Vorbehalt weist lediglich darauf hin, dass der Umfang der vorgestellten Wortliste nicht endgültig sein muss.

\section{Kriterien für die Auswahl von Belegen}

Die unten angeführte Wortliste stellt eine Auswahl von entlehnten Substantiven dar, die anhand des Idiolekts des Artikelautors zusammengestellt wurde. Sie erfolgte mehr oder 
weniger in drei Schritten: 1. Auswahl der Entlehnungen und ihre primäre Einteilung im Einklang mit dem Idiolekt und Sprachgefühl des Autors; 2. Überprüfung und Präzisierung nach SSJČ (1989) und NEWERKLA (2004); 3. Vergleich mit dem Korpus SYN 2000 (öffentlich zugänglicher Teil). Außer Acht gelassen wurde die Beschreibung von entlehnten Verben, Verbgefügen, Phraseologismen und Partikeln. Als Quelle der Belege galt für uns in erster Linie die Allgemeinsprache, obwohl die Fachsprache und insbesondere die Fachjargons eine weitaus größere Menge von deutschen Entlehnungen ahnen lässt. Aus der Fachsprache wurden nur solche Wörter übernommen, die ganz allgemein verständlich sind und verhältnismäßig häufig gebraucht werden wie z. B. šaltrpáka ,Schalthebel', blinkr ,Blinker', hever ,Heber', šmirglpapir ,Schmirgelpapier" usw. Bei der Klassifizierung des entlehnten Wortguts war für uns die Zugehörigkeit der Belege zu einer Sprachebene und die territoriale Verbreitung entscheidend. Keine Beachtung wird der Beschreibung der Wortbildungsart sowie der Konstituentenanalyse innerhalb eines entlehnten Worts geschenkt.

\section{Bedeutungsverschiebung bei der Übernahme des fremden Wortguts}

Aus dem Vergleich des Ausgangsformativs mit der Entlehnung geht hervor, dass sich die Bedeutungen nicht immer hundertprozentig decken. Gemeint ist dabei die Hauptbedeutung des Denotats in standardsprachlichen Ausdrücken. Das entlehnte Lexem ešus geht auf Essschüssel zurück. Es ist jedoch in der Nehmersprache keine ,Essschüssel' im Sinne ,jídelní miska', sondern ein ,kompaktes Essgeschirr für unterwegs' (beim Militär, Camping oder Picknick). Wichtig ist dabei, dass der Deckel abnehmbar und zugleich die ganze Vorrichtung zum Aufhängen ist. Eine Umschreibung des so gestalteten Denotats lautet ,souprava jídelních misek pro polní stravování (s. http://cs.wikipedia.org). Als Warenposten in einem Warenkatalog wird jedoch eindeutig die Bezeichnung ěsus bevorzugt (s. http://nadobi.hledej.cz). Dem deutschen Lexem Macher in der Bedeutung, jemand, der sich durch große Tatkraft, Durchsetzungskraft, durch die Fähigkeit zum Handeln auszeichnet', entspricht im Tschechischen machr in der Bedeutung ,geschickter Mensch, hervorragender Fachmann' mit den Zusatzmerkmalen gs. expr. Im Tschechischen kann dieses Lexem auch die Markierung, abwertend' annehmen und es wird dann dadurch ein Mensch bezeichnet, der überheblich auftritt und alles kann und weiß. In SSJČ (1989) ist nur das entsprechende Verb machrovat (gs. abw.) im Sinne ,angeben, prahlen belegt. Die Entlehnung rychtár im Sinne ,Gemeindevorsteher, Bürgermeister ' (s. NEWERKLA: 2004, 139 f.) wird im Tsch. als veraltet und gs. empfunden (s. SSJČ V: 1989, 213). Das ahd. Ausgangsformativ rihtâri weist die Bedeutung ,Leiter, Richter' auf. (s. PFEIFER: 1989, 1425). Die Extension des entlehnten Lexems handlir ist gegenüber dem Lexem in der Gebersprache Händler als ,Vieh- bzw. Pferdehändler eingeschränkt. Darüber hinaus ist das Wort als historischer Ausdruck in der Gemeinsprache gekennzeichnet (s. SSJČ II: 1989, 11). Eine sichere deutsche 
Herkunft weist zweifelsohne das tschechische Lexem hajzl auf. Es kann entweder als Schimpfwort ,schlechter, mieser Kerl' oder als Bezeichnung eines ,Aborts', in beiden Fällen mit dem Merkmal vulg., verwendet werden (s. SSJČ II: 1989, 6). Das Motiv für die vulgäre Bezeichnung der Bedürfnisanstalt ist in dem südd. Diminutiv Häusel mit anschließender Entrundung zu suchen. Es handelt sich eigentlich um eine überdachte Latrine im Hof eines bäuerlichen Anwesens, die äußerlich an das Aussehen eines Häuschens erinnert.

\section{Deutsche Entlehnungen in der Standardsprache (Schriftsprache)}

Als Kriterium für die Zuordnung zur Standardsprache galt Folgendes: Für die Entlehnung gibt es keine tschechische Entsprechung (in den meisten Fällen eine Einwortbezeichnung), die sich semantisch mit der Entlehnung völlig decken würde. Die Entlehnung ist auf dem ganzen Territorium Tschechiens verbreitet und verständlich. Es ist dabei nicht ausgeschlossen, dass sich diese Entlehnungen weiter differenzieren lassen, und zwar in Bezug auf zeitliche Zuordnung, soziale Zugehörigkeit und andere Gebrauchsangaben wie ,Schimpfwort', ,abwertend' usw.

Es sind hier z. B. folgende Fälle zu nennen: fracek (nach SSJČ I: 1989, 518, mit dem Merkmal ,derb') = Fratz (österr./südd. ,ungezogenes Kind, besonders Mädchen'), fuchtle (nach SSJČ I: 1989, 526 ,Dirne', vulg. oder Schimpfwort für die Frau allgemein) = Fuchtel (österr./südd. ,zänkische, herrschsüchtige Frau'), hokynár (nach SSJČ II: 1989, 62 mit dem Merkmal ,früher ${ }^{r}$ ) = Höker (Kleinhändler, veraltet). Mit dem Suffix -stvíwird dazu das Substantiv hokynárstvíabgeleitet (s. SSJČ II: 1989, 62) mit der Bedeutung ,kleiner Laden mit Nahrungsmitteln und anderen Waren'. Im Deutschen hat die Ableitung keine Entsprechung. Als veraltet bewertet SSJČ II $(1989,431)$ das Lexem kramár $=$ (Krämer, Kramer, landsch.). Ferner gehört hierher das Schimpfwort ludra (SSJČ III: 1989, 585 führt die Variante ludr an = Luder (salopp, ,durchtriebener, gemeiner Mensch') und pakáž (in SSJČ IV: 1989,11 als abwertend gekennzeichnet) = Pack (salopp, abwertend). Das entlehnte Wort špejle (s. SSJČ VI: 1989, 60) wird nicht expressiv verwendet.

Ein gewisses Problem stellen die Ausdrücke fešák (SSJČ I: 1989, 493) = fescher Kerl, Feschak, (österr. ugs.) und špehýrka (SSJČ VI: 1989, 59) = Spähloch dar. Sie können kaum durch ein schriftsprachliches Äquivalent ersetzt werden. Trotzdem wird in SSJČ (1989) im ersten Fall das Merkmal gs. und im zweiten Fall das Merkmal ats. angeführt. Aus der Sicht der Wortbildung ist zu erwähnen, dass bei špehýrka nur das Erstglied der Zusammensetzung ,Spähloch` übernommen wird, das Zweitglied wird durch das tschechische Suffix -irka ersetzt. Im Gegensatz dazu ist der Ausdruck haksna mit dem stilistischen Merkmal vulg. (s. SSJČ II: 1989, 7) gekennzeichnet. Weil eben ein neutrales Äquivalent in der Schriftsprache als noha existiert, neigen wir dazu, diesen Ausdruck eher auf der Ebene des alltagssprachlichen Standards anzusiedeln. 
Das Ende der Entstehungszeit des SSJČ (1989) ist mit den achtziger Jahren des vorigen Jahrhunderts verbunden. Es liegt deswegen auf der Hand, dass neuere Entlehnungen nicht verzeichnet sind. Wir können z. B. das für die frühere Gesellschaftsordnung so typische Wort vekslák mit dem Merkmal abw. anführen. Es geht um eine Person, die den Ausländern auf der Straße Geldumtausch anbietet bzw. aufzwingt. Die Entlehnung geht auf das Substantiv Wechsler in der Bedeutung ,Person, die beruflich in einer Wechselstube Geld wechselt' zurück. SSJČ (VII: 1989, 41) kennt nur das Stichwort veksl bzw. das abgeleitete Verb vekslovat in vier anderen Bedeutungen, die entweder als veraltet bzw. als Fachslang einzustufen sind. Im Sportjargon und in der Publizistik setzt sich immer mehr die Bezeichnung šsilmachr durch. Bezeichnet wird dadurch der Spieler, der das Spiel seiner Mannschafft entscheidend bestimmt (Spielmacher).

\section{Deutsche Entlehnungen in der Gemeinsprache}

Als gemein- bzw. umgangssprachlich haben wir solche Lexeme bezeichnet, die in der gesprochenen Alltagssprache gebräuchlich sind und zu denen es keine Entsprechungen in der Standardsprache (Schriftsprache) gibt. Es handelt sich um eine lockere Sprechweise, die kaum gegenüber einem gesellschaftlich höher gestellten Gesprächspartner verwendet werden kann. Sie wird vom Sprecher unter den sozial Gleichgestellten mit dem Ziel gebraucht, sich von dem üblichen Sprachusus in bestimmtem Maße abzuheben, der eigenen Ausdrucksweise mehr Nachdruck zu verleihen, den eigenen Idiolekt mehr in den Vordergrund zu stellen. Diese stilistische Schicht kann mit folgenden Beispielen demonstriert werden:

aušus (SSJČ I: 1989, 61) ,vadný výrobek, zmetek' = Ausschuss (minderwertige Ware), dekl (SSJČ I: 1989, 290) ,víko' = Deckel, foch (SSJČ I: 1989, 508) ,přihrádka ${ }^{\prime}=$ Fach (zum Aufbewahren von etwas), futro (SSJČ I: 1989, 530) ,dveřní rám', ,podšívka' = Futter (Holzauskleidung, Stoff auf der Innenseite von Kleidungsstücken), hever (SSJČ II: 1989, 29) ,zdvihák, zvedák' = Wagenheber, ruksak (SSJČ V: 1989, 108) ,batoh ‘ = Rucksack, rýgl (SSJČ V: 1989, 210) ,zástrčka ‘ = Riegel (Vorrichtung zum Verschluss von Türen), šichta (SSJČ VI: 1989, 23) ,směna ${ }^{\prime}=$ Schicht (Arbeitsschicht), šiml (SSJČ VI: 1989, 25) ,bělouš ${ }^{\prime}=$ Schimmel (Pferd), šminka (SSJČ VI: 1989, 51), líčidlo‘ = Schminke, šmirglpapir (SSJČ VI: 1989, 52) ,smirkový papír ${ }^{`}=$ Schmirgelpapier, špagát (SSJČ VI: 1989, 57), ,provaz‘ = Spagat (südd./österr.), špajzka/špajz (SSJČ VI: 1989, 57), ,špižírna/špížka' = Speis (südd./österr. ugs.), šraňk (SSJČ VI: 1989, 69) ,závora' $=$ Schranke, štrúdl (SSJČ VI:

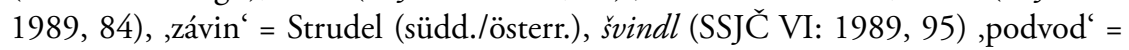
Schwindel (Betrug).

Eine Sonderstellung nimmt m. E. die Entlehnung fusak = Fußsack ein. SSJČ (1989) verzeichnet diesen Ausdruck nicht. Bei NEWERKLA (2004, 440) steht fusak als ein Ausdruck im Slowakischen der niedrigeren Alltagssprache. Auf Grund 
meines Idiolekts würde ich diesen Ausdruck auch in die tschechische Gemeinsprache einordnen. Im Korpus SYN 2000 ist diese lexikalische Einheit nur einmal belegt, die niedrige Vorkommenshäufigkeit ist auf die fachliche Spezifik des Ausdrucks zurückzuführen. Eine schriftsprachliche Entsprechung lautet ,nánožník‘.

\section{Deutsche Entlehnungen in der Gemeinsprache mit Konnotationen}

\subsection{Zeitliche Dimension}

In manchen Fällen führt SSJČ weitere Zusatzmerkmale an, die die zeitliche Dimension und die Expressivität kennzeichnen. Die folgenden lexikalischen Einheiten werden in SSJČ (1989) mit dem Merkmal ,veraltet' ergänzt: erteple, ,brambory' (SSJČ I: 1989, 463) = Erdäpfel (österr. ugs.), gábl,přesnídávka' (SSJČ I: I989, 532) = Gabelfrühstück (veraltend, ,zweites Frühstück bei besonderen Anlässen eingenommen'), kvér, ,puška' (SSJČ II: 1989, 493) = Gewehr, oringle, ,náušnice‘ (SSJČ III: 1989, 583) = Ohrringe, špacírka, vycházková hůl' (SSJČ VI: 1989, 57) = Spazierstock, tapecír, ,čalouník' (SSJČ VI: 1989, 118) = Tapezierer, kvelb, ,obchod“ (SSJČ II: 1989, 493) = Gewölbe, lont, ,venkov' (SSJČ II: 1989, 576) = Land.

Durch das Merkmal ,einigermaßen veraltet' zeichnen sich nach SSJČ (1989) folgende Lexeme aus: fusekle (SSJČ I: 1989, 530) = Fußsocken, grif, hmat' (SSJČ I: 1989, 551) = Griff, kysna, ,bedna' (SSJČ II: 1989, 502) = Kiste, luft, ,vzduch ‘ (SSJČ II: 1989, 586) = Luft, piksla, ,plechovka' (SSJČ IV: 1989, 103) = Büchse, plac ,místo' (SSJČ IV: 1989, 116) = Platz, raubiř ,loupežník' (SSJČ V: 1989, 24) = Räuber, šnycl ,ř́zek‘ (SSJČ VI: 1989, 54) = Schnitzel, štrozok, ,slamník‘ (SSJČ VI: 1989, 84) = Strohsack.

Ausgehend vom Idiolekt des Artikelautors ist die Menge der oben genannten Beispiele auf folgende Fälle zu reduzieren: erteple, špacirka, tapecír, oringle, raubir. Dass die Angaben über die zeitliche Einordung der Belege zu relativieren sind, bezeugt auch die festgestellte Vorkommenshäufigkeit der betreffenden Einheiten im Korpus SYN 2000 (öffentlich zugänglicher Teil). In Übereinstimmung mit den Angaben von SSJČ (1989) sind selten zu verzeichnen: kysna, gábl, oryngle, raubiŕ, šnycl, tapecír, špacirka, lont und strozok. Die anderen Ausdrücke, die in SSJČ (1989) als veraltet bzw. als einigermaßen veraltet gekennzeichnet sind, sind in den in das Korpus eingespeicherten Texten verhältnismäßig häufig anzutreffen: erteple (7x), fusekle (23x), grif (16x), kvér (21x), luft (13x), piksla (8x), plac (81x), kvelb $(18 \times)$.

\subsection{Expressivität}

Die Expressivität wird in den angeführten Entlehnungen entweder als abwertend: kripl, ,mrzák' (SSJČ II: 1989, 442) = Krüppel oder als derb spezifiziert: fotr, ,otec (SSJČ I: 1989, 516) = Vater, ksicht, ,obličej' (SSJČ II: 1989, 470) = Gesicht, 
mutra, ,matka' (SSJČ III: 1989,154) = Mutter, kušna, ,huba' (SSJČ II: 1989, 487) = Gosche/Gusche (besonders südd./österr. meistens abw.). Im Korpus SYN 2000 kommen diese Ausdrücke mit Ausnahme von kušna sehr häufig vor. Die Entlehnung štamgast, stálý host' (SSJČ VI: 1989, 71) wird zu der alltagssprachlichen Ebene mit den Zusatzmerkmalen ,expressiv und einigermaßen veraltet' zugeordnet. Im Korpus SYN 2000 kommt das Wort jedoch $24 \times$ vor.

Im Fall der Stichwörter švigrfotr, ,tchán', švigrmutr, ,tchyněc (SSJČ VI: 1989, 94; NEWERKLA: 2004, 509) gehen die Quellen in der Bewertung der Konnotationsmerkmale einigermaßen auseinander. Während SSJČ (1989) die Merkmale ,allgemeinsprachlich' und ,veraltet' anführt, führt NEWERKLA (2004) das Merkmal vulg. an. Wir sind der Meinung, dass beide Werke Recht haben, und zwar in dem Sinne, dass das Lexem einerseits als historischer Ausdruck dient und zugleich in der Gegenwartssprache aktiviert wurde, allerdings mit der bei NEWERKLA (2004) erwähnten Expressivität, die bei švigrmutr mit der Variante švigrmutra eindeutig zum Ausdruck gebracht wird.

\section{Nichtschriftsprachliche Entlehnungen}

\subsection{Slangausdrücke deutscher Herkunft}

Folgende Beispiele sind als lexikalische Einheiten zu betrachten, die vor allem für die Ausdrucksweise verschiedener Berufsgruppen bzw. Interessengemeinschaften kennzeichnend sind. Ziemlich allgemein verbreitet sind nach SSJC (1989) die Slangausdrücke ajznpoňák, ,̌̌elezničář (SSJČ I: 1989, 14) = Eisenbahner, cimra, ,pokoj‘ (SSJČ I: 1989, 211) = Zimmer, vercajk ,nářadí‘ (SSJČ VII: 1989, 58) = Werkzeug. Ferner gehört hierher fachman ,odborník‘ (SSJČ I: 1989, 480) = Fachmann und nášup ,př́ídavek jídla při hromadném stravování (SSJČ III: 1989, 269; NEWERKLA: 2004, 472) = Nachschlag, Nachschub (Versorgen von Truppen mit Nahrungsmitteln). Eine tschechische Entsprechung in einem tschechischen Satz muss mit dem Verb pridat = ,dazugeben' umschrieben werden: ,Wer will einen Nachschlag? Kdo chce prìdat?` Die große Vorkommenshäufigkeit der angeführten Slangausdrücke lässt sich auch aus dem Korpus SYN 2000 bestätigen. Eine Ausnahme ist das Wort ajznpoñák, das in den Texten nur einmal vorkommt. Dies ist wohl auf das zu fachspezifische Denotat zurückzuführen.

\subsection{Mundartliche Ausdrücke deutscher Herkunft}

Für mundartlich gekennzeichnete Ausdrücke gelten nach SSJČ (1989) z. B. flaška, ,láhev' (SSJČ I: 1989, 503) = Flasche, kél ,kapusta' (SSJČ II: 1989, 308) = Kohl und šopa ,kůlna' (SSJČ VI: 1989, 54) = Schuppen. Bei flaška führt SSJČ (1989) noch die zeitliche Zuordnung als, veraltet' an. Dieser Zuordnung widerspricht allerdings der Korpusbefund, wo dieses Wort $43 \times$ belegt ist. Demgegenüber ist das Wort kél 
im Korpus SYN 2000 nicht enthalten, šopa kommt nur zweimal vor, und zwar als Bestandteil des Fluchs kurnik šopa.

\title{
7.3. Territorial beschränkter Usus deutscher Entlehnungen - die alltägliche Umgangssprache der Stadt Brno
}

Manche Entlehnungen sind nicht ganz allgemein in Tschechien verbreitet, sondern ihre Verwendung ist vorwiegend auf Brno beschränkt. Wir sind nicht der Annahme abgeneigt, dass die Bevorzugung deutscher Entlehnungen mit der sozialen Differenz der Stadtbewohner, ihres Alters und des Stadtgebietes zusammenhängen könnte, wie dies übrigens KRČMOVÁ (1981, 181 f.) im Falle der lautlichen und morphologischen Besonderheiten der alltäglichen Umgangssprache in Brno bestätigt hat. Ein signifikanter Hinweis auf die territoriale Zugehörigkeit der unten angeführten Entlehnungen könnte der Umstand sein, dass sie bis auf eine Ausnahme (s. facha ,Arbeit' bei NEWERKLA: 2004, 431) weder in SSJČ (1989) noch bei NEWERKLA (2004) enthalten sind. Ohne weitere Zusatzmerkmale sind hier z. B. folgende zu nennen: fišla ,ryba ${ }^{\prime}=$ Fisch, fórrichtuňk ,zařízení, přístroj ${ }^{\prime}=$ Vorrichtung, gajgle ,housle $^{\prime}=$ Geige, krempl, zmetek, krám ${ }^{\prime}=$ Krempel (ugs. abw. Sachen, die nicht viel wert sind oder als solche angesehen werden), krýgl, pivní sklenice $^{\prime}=$ Krügel (ein südd. Diminutiv zu Krug), lochec ,smích, řehot ${ }^{\prime}=$ Lachen, perza , peněženka ${ }^{\prime}=$ Geldbörse (mlat. bursa im Sinne ,Lederbeutel', lat. byrsa = ,abgezogene Haut, Fell'), statl, město ${ }^{\prime}=$ Stadt.

Einige der Entlehnungen sind wahrscheinlich nur bei der älteren Generation in Gebrauch, wie dies in den folgenden Beispielen der Fall ist: ancuk, oblek' $=$ Anzug, $k o ́ d r$, prsten $^{`}=$ Ring (Kleinod, ,Köder ${ }^{`}$ wird hier im übertragenen Sinn und abwertend als etwas, was als Lockmittel benutzt wird, verwendet). Die folgenden Ausdrücke können zusätzlich durch das Merkmal ,salopp' charakterisiert werden: glocna ,pleš, hlava $^{`}=$ Glatze, hicna , horko ${ }^{\prime}=$ Hitze (SSJČ II: 1989, 30 und NEWERKLA: 2004, 445 führen nur hic an), hokna ,práce $^{c}=$ Hocken (Arbeit, südd.), kaltna ,zima,

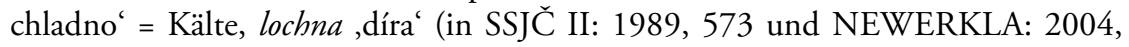
198 nur loch), rychna smrad $^{\prime}=$ Geruch. Die Existenz der präsentierten territorial beschränkten Entlehnungen bestätigt auch der Korpus SYN 2000 (öffentlich zugänglicher Teil), wenn auch nur in sehr begrenztem Umfang. Verzeichnet sind folgende Lexeme, und zwar im Kontext der Umgangssprache in Brno: krýgl, lochec, statl, kaltna. Den territorialen Rahmen sprengt offensichtlich facha $(3 \times)$ und háro , vlasy' $=$ Haar $(3 \times)$.

\author{
Abkürzungsverzeichnis: \\ abw. abwertend \\ ahd. althochdeutsch \\ ats. Alltagssprachlich \\ BMK Brněnský mluvený korpus
}




$\begin{array}{ll}\text { ČNK } & \text { Český národní korpus } \\ \text { expr. } & \text { expressiv } \\ \text { gs. } & \text { gemeinsprachlich } \\ \text { österr. } & \text { österreichisch } \\ \text { südd. } & \text { süddeutsch } \\ \text { SYN } & \text { Synchronní korpus českého jazyka } \\ \text { ugs. } & \text { umgangssprachlich } \\ \text { vulg. } & \text { vulgär }\end{array}$

\section{Zusammenfassung:}

Die vorhandene Übersicht über die deutschen Entlehnungen aus stilistischer Sicht hat angedeutet, dass das entlehnte Wortgut nicht nur in der Standardsprache Fuß fasst, sondern auch in niedrigeren Stilschichten, nicht selten von weiteren konnotativen Merkmalen begleitet, einen Platz einnimmt. Diese Erscheinung stellt dabei nichts Einmaliges dar. Vielmehr handelt es sich hier um eine universelle Tendenz, die darauf beruht, dass Sprachen im Allgemeinen einen großen Bedarf an neuen und expressiven Ausdrücken haben. Und im Falle des Tschechischen wird dieser Bedarf durch deutsche Entlehnungen gedeckt (vgl. auch NEWERKLA: 2004, 77).

Eine Gegenüberstellung der hier behandelten Entlehnungen mit den Wörterbucheintragungen SSJČ (1989) und NEWERKLA (2004) sowie auch mit dem Korpus SYN 2000 hat zweierlei gezeigt: Nicht alle vorgestellten Entlehnungen sind in den verwendeten Quellen enthalten. Dies betrifft vor allem diejenigen, die aus niedrigeren Sprachebenen stammen. Eine gewisse Diskrepanz zeigt sich zwischen den in Wörterbüchern aufgezeichneten Zusatzmerkmalen auf der einen Seite und den dem Korpus SYN 2000 entnommenen Angaben auf der anderen Seite. Hier ließe sich durch Befragungen von Gewährsleuten aus verschiedenen sozialen Schichten, Altersgruppen und Gebieten mit Hilfe von Fragebögen, wie dies auch SKÁLA (1968, 127-141) praktiziert, effektiv Abhilfe schaffen. Nur so könnten alle vorhandenen Entlehnungen erfasst und stilistisch überprüft werden, die sonst durch andere Methoden (spontane Sprechäußerungen, gelenkte Gespräche usw.) kaum ins Blickfeld rücken würden.

Schlüsselwörter:

Entlehnungen - niedrigere Sprachebenen - Befragungen - Wörterbucheintragungen Stilschichten - Standardsprache

\section{Literatur:}

HUGO (2006): Hugo, Jan. Slovnik nespisovné češtiny. Praha: Maxdorf, 2006.

KRČMOVÁ (1981): Krčmová, Marie. Běžně mluvený jazyk v Brně. Brno: Masarykova univerzita, 1981.

MIČKA (2007): Mička, Pavel. Germanismen im Wörterbuch des nichtschriftsprachlichen Tschechischen. Brno: Masarykova univerzita. Pedagogická fakulta, Dipl. 2007.

MUZIKANT (2002): Muzikant, Mojmír. Sprachatlanten und die deutsch-tschechischen Sprach- kontakte auf lexikalischer Ebene, in: Zeitschrift für Dialektologie und Linguistik, 2002, 69, Nr. 2, 182-188.

NEWERKLA (2004): Newerkla, Stefan Michael: Sprachkontakte Deutsch - Tschechisch Slowakisch. Frankfurt am Main: Peter Lang, 2004. 
PFEIFER (1989): Pfeifer, Wolfgang und Koll. Etymologisches Wörterbuch des Deutschen. Berlin: Akademie-Verlag, 1989.

SKÁLA(1968): Skála, Emil. Deutsche Lehnwörter in der heutigen tschechischen Umgangssprache, in: Havránek, Bohuslav/Fischer, Rudolf (Hg.): Deutsch-tschechische Beziehungen im

Bereich der Sprache und Kultur: Aufsätze und Studien. Berlin: Akademie-Verlag, 1968, 127-141.

SSJČ (1989): Slovnik spisovného českého jazyka. Praha: Academia, 1989. 